\title{
Progress of the patients with diabetes mellitus who were managed with the staged diabetes management framework*
}

\author{
Evolução do tratamento de pacientes diabéticos utilizando o protocolo staged diabetes management
}

Evolución del tratamiento del paciente diabético utilizando el protocolo staged diabetes management

\begin{abstract}
Objective: To describe the progress of patients with diabetes mellitus seen by health care team members who followed the Staged Diabetes Management framework. Methods: This descriptive, prospective, and longitudinal study was conducted in a period of 12 months. The sample consisted of 54 patients with diabetes mellitus. Data were collected in three occasions through interviews: P0 - at beginning of the study; P6 - in six months; and, P12 - at the end of the study. Results: There was an increase in adherence to diet from $61.1 \%$ in the P0 to $92.6 \%$ in the P12, in adherence to physical activities from 57.4\% in the P0 to 66,7\% in the P12, and in the use of medication. Conclusion: The use of the SDM framework among this sample of patients with diabetes promoted better adherence to diet, physical activities, and the use of medication.
\end{abstract}

Keywords: Diabetes mellitus; Clinical protocols; Patient compliance; Nursing

\section{RESUMO}

Objetivo: Descrever a evolução do tratamento de pacientes diabéticos por equipe multiprofissional utilizando o Protocolo Staged Diabetes Management. Métodos: Estudo descritivo, prospectivo e longitudinal, realizado em 12 meses com pacientes diabéticos ( $\mathrm{n}=54$ ). Os dados foram coletados mediante entrevista em três pontos: P0 - início do estudo; P6 - seis meses após o início; e P12 - final do estudo. Resultados: Houve aumento no cumprimento do plano alimentar pelos pacientes diabéticos, de $61,1 \%$ no P0 para 92,6\% no P12. No que se refere à atividade física, observou-se aumento de 57,4\% no P0 para 66,7\% no P12. Verificou-se também um aumento da cobertura medicamentosa no tratamento do diabetes. Conclusão: A utilização do Protocolo Staged Diabetes Management por equipe multiprofissional aumentou a cobertura medicamentosa no tratamento para o diabetes e a adesão ao plano alimentar e atividade física pelos pacientes diabéticos.

Descritores: Diabetes mellitus; Protocolos clínicos; Cooperação do paciente; Enfermagem

\section{RESUMEN}

Objetivo: Describir la evolución del trataminto de pacientes diabéticos por un equipo multiprofesional utilizando el Protocolo Staged Diabetes Management. Métodos: Se trata de un estudio descriptivo, prospectivo y longitudinal, realizado en 12 meses con pacientes diabéticos $(\mathrm{n}=54)$. Los datos fueron recolectados mediante entrevista en tres puntos: P0 - inicio del estudio; P6 - seis meses después del inicio; y P12 - final del estudio. Resultados: Hubo aumento en el cumplimiento del plan de alimentación por los pacientes diabéticos, del 61,1\% en el P0 y del 92,6\% en el P12. En lo que se refiere a la actividad física, se observó aumento del 57,4\% en el P0 y del 66,7\% en el P12. Se verificó también un aumento de la cobertura medicamentosa en el tratamiento de la diabetes. Conclusión: La utilización del Protocolo Staged Diabetes Management por un equipo multiprofesional aumentó la cobertura medicamentosa en el tratamiento para la diabetes y la adhesión al plan de alimentación y actividad física por los pacientes diabéticos.

Descriptores: Diabetes mellitus; Protocolos clínicos; Cooperación del paciente; Enfermería

\footnotetext{
* Study carried out Education Center the Nursing to Adults and Elders in College of Nursing in Ribeirão Preto the University of São Paulo - USP.

1 Associate Professor at the University of Sao Paulo at Ribeirao Preto College of Nursing USP-Ribeirão Preto (SP)- Brazil.

${ }^{2}$ DNS (Fundamental Nursing) by University of Sao Paulo at Ribeirao Preto College of Nursing USP- Ribeirão Preto (SP)- Brazil.

${ }^{3}$ Psychologist, MMS by the University of Sao Paulo at Ribeirao Preto College of Medicine - USP-Ribeirão Preto (SP)- Brazil.

${ }_{5}^{4}$ DNS, Professor at the University of Sao Paulo at Ribeirao Preto College of Philosophy, Sciences and Languages - USP-Ribeirão Preto (SP)- Brazil.

${ }^{5}$ Nutritionist; MNS (Public Health) by the University of Sao Paulo at Ribeirao Preto College of Nursing USP- Ribeirão Preto (SP)- Brazil.

${ }^{6}$ DNS, Professor at University of Sao Paulo at Ribeirao Preto College of Medicine USP-Ribeirão Preto (SP)-Brazil.
} 


\section{INTRODUCTION}

Education has been recommended as an essential aspect in diabetes treatment. Moreover, several studies have demonstrated its importance in different communities with specific socioeconomic and cultural characteristics ${ }^{(1-2)}$. In this view, an effective diabetes education program requires training, knowledge, pedagogical skills, ability to communicate and listen, understanding, and negotiation skills from the multiprofessional health team ${ }^{(2)}$.

The need to develop educational activities or educational health practices for diabetic patients and their families is associated with preventing complications by means of diabetes self-management, which enables patients to better cope with the disease ${ }^{(3-4)}$. In this sense, the multiprofessional team has the role to provide patients with all the information regarding the illness, as well as to follow them for a certain period with a view to help them make decisions in face of the numerous diabetesrelated situations.

Despite the recommendations for diabetes education, few studies have effectively evaluated diabetes education program results. To provide such effective evaluations, data must be obtained both before and after the intervention ${ }^{(5)}$. It is worth emphasizing that approximately one-forth of researchers find it difficult to evaluate intervention effectiveness. On the other hand, diabetes education is effective when offered systematically, through consistent programs and within a determined period.

In acknowledgement of the need for studies of this particular nature, the present work was developed to follow diabetic patient treatment evolution concerning diet, physical activities, and drug treatment. Patients were followed by a multiprofessional health team, at a center for research and continuing education using the Staged Diabetes Management protocol.

\section{STAGED DIABETES MANAGEMENT - SDM}

SDM is a protocol for systematic healthcare of diabetic patients, developed by the International Diabetes Center, in Minneapolis (USA) in 1989. It comprises one textbook and two Fast Guides for diabetic patient care. The Fast Guides are documents based on recommendations by the American Diabetes Association, the National Diabetes Data Group, the International Diabetes Federation, and the World Health Organization. They delineate treatment foundations and the decision-making methods regarding treatment. They also guide the multiprofessional team in providing diabetic patients with the pathways to manage their disease. This guide is structured as to classify and diagnose the disease, to outline treatment options and control metabolic and lipid parameters, using an algorithm that establishes the maximum time for each type of intervention in the initial, adjustment, and maintenance treatment phases.

The Guide comprises three treatment stages. The nutritional stage comprises providing diet advice and the implementation of a physical activity program to help patients achieve their metabolic goals. The stage referred to as oral agents concerns the use of oral hypoglycemic agents. Finally, the insulin stage involves managing fast-, intermediate-, and long-action insulin.

The treatment stages indicate which type of treatment should be selected for the patient. For each treatment stage, patients go through three treatment phases: the initial phase, the adjustment phase, and the maintenance phase; considering that the diabetic patient undergoes a process, which starts with the diagnosis and evolves to the adjustment phase until the goals are achieved. At this point, treatment is maintained. Hence, the diabetic patient care is defined through the treatment stages that indicate the expected progresses.

In the initial phase, the multiprofessional team obtains patient data for diagnosis and to begin the treatment. It is worth stressing that each type of diabetes or complication requires different information and data for diagnosis and making clinical decisions. Changes are made during the treatment adjustment phase. These treatment adjustments have the purpose to optimize illness control. This phase can take days or months and is founded on patients' substantial participation, since changes are made upon the data they provide.

The maintenance phase begins when patients reach the expected goals and are actively involved in treatment maintenance. It is expected that patients enter and leave this phase regardless of the treatment stage they are in. Various factors affect patients' leaving this phase: changes in lifestyle, compliance to the diet plan, social and psychological adjustments to the illness, the desire to achieve good control and natural diabetes progression.

SDM recommends that stages and the respective phases be implemented through the development of an educational program directed to the patients' real needs. The treatment stages are adjusted to each kind of diabetes, and can be introduced, adjusted, or replaced throughout the treatment. Therefore, they are dynamic. This protocol has been used in several countries, and evidenced a reduction in the levels of Hemoglobin A1c as well as in diabetes-related chronic complications ${ }^{(6)}$. In the United States, large health care centers determined nurses would administrate SDM. In these centers, nurses, as SDM managers, provided health care, education, and nutrition services to diabetic patients. They used the adjusted Fast Guides together with the patient and a primary health care physician to determine the metabolic control goals. When goals were not reached, nurses had the autonomy 
to change the drug dose, using the algorithms proposed in the SDM Fast Guides. In five health institutions, patient metabolic control was improved when nurses were the ones responsible for $\mathrm{SDM}^{(1)}$. In Brazil, nurses' lack of professional autonomy and preparation concerning diabetes are factors that make it difficult to use this kind of protocols.

\section{PURPOSE}

To describe treatment evolution regarding the diet plan, physical activity, and drug therapy of diabetic patients assisted by a multiprofessional team, using the SDM protocol.

\section{METHODS}

This is a descriptive, prospective and longitudinal study, performed from April 2004 to April 2005, at a center for research and continuing education in the city of Ribeirao Preto, Sao Paulo State, Brazil. The staff responsible for following patients was coordinated by an advance practice nurse, who was responsible for the diabetes education program at this center, in addition to three nurses, two endocrinologists, one nutritionist, and three psychologists.

The study population consisted of 240 patients with type 1 and type 2 diabetes. Patients were men and women from Ribeirao Preto and neighboring citied, who were registered at the center and agreed to participate in the study. The patients' diabetes diagnosis was confirmed by means of fasting plasma glucose test. Patients were excluded if: they had gestational diabetes; they had an incomplete register at the center; their address was not found; they refused to participate in the study; they reported having difficulties to participate due to work or school; they reported to be currently participating in another program offered by their health insurance; and, evidently, those who were deceased. Hence, after establishing the inclusion and exclusion criteria, the study population consisted of 57 patients with type 1 and type 2 diabetes. During this study, two patients died and one abandoned the program; so 54 patients took part in the study.

Data collection was performed using a questionnaire proposed by SDM, containing three questions about the treatment concerning the diet plan, physical activity, and drug therapy. The first and second questions refer to complying with the diet plan and physical activity, respectively. These questions asked patients to answer if they had been following the recommendations received from the multiprofessional team regarding the prescribed diet plan and suggested physical activity. The third question was split into two parts: one regarding taking oral hypoglycemic agents, and the other about taking insulin, in monotherapy and/or in combination. This question also asked patients to state the name, dose, and hours of the drugs being used to control diabetes.

The interview for data collection was performed before medical, nursing, and nutritional appointments in three moments: $\mathrm{P} 0$ - beginning of the study; $\mathrm{P} 6$ - six months later; and P12 - end of the study. It should be stressed that these patients were being followed by the Diabetes Education Group, participating in the SDM Research Protocol, and that the treatment conducts of the team following these diabetic patients in terms of the diet plan, physical activity, and drug therapy were based on the decision algorithms proposed by the SDM Protocol, going through several treatment stages and phases, according to each patient's needs.

To organize the data, a database was created using SPSS 11.5; data was double typed. The results were presented using absolute frequencies and percentages. The project was approved by the Institutional Review Board at the University of Sao Paulo at Ribeirao Preto College of Nursing, under protocol number 03172002.

\section{RESULTS}

The study population consisted of 54 adult and elderly patients, with ages between 29 and 78 years (average 60 years); $74.1 \%$ were women. Regarding the type of diabetes, most (96.3\%) had type 2; the average diagnosis time was 6 years; the most common comorbidities were hypertension, obesity, dyslipidemia, and vascular problems.

Table 1 shows the numerical and percentile evolution of patients assisted at the referred center, according to the diabetes treatment.

It is observed that diabetic patients' compliance with the diet plan increased after implementing the SDM, since there was a progressive increase from $61.1 \%$ who complied at P0 to $92.6 \%$ at P12. This indicates the importance that patients ascribe to their diets. Regarding physical activity, a more modest increase was observed, that is, from $57.4 \%$ at $\mathrm{P} 0$ to $66.7 \%$ at $\mathrm{P} 12$.

In terms of the drug therapy, at $\mathrm{P} 0,65.1 \%$ of the 54 diabetic patients reported taking oral hypoglycemic agents, of which $24.1 \%$ were Biguanides, and 20.4\% combined Biguanides and Sulfonylureas. It is observed that at P12 there was an increase in the number of patients taking Biguanides as monotherapy and the combination of Biguanides with Sulfonylureas. It is also observed that fewer patients took Sulfonylureas as a monotherapy. Of the $31.5 \%$ who reported taking insulin at $\mathrm{P} 0,27.8 \%$ used NPH insulin and $3.7 \%$ the combination of NPH and regular insulin. It is observed that at $\mathrm{P} 6$ there was an increase in the number of patients 
taking insulin, to $35.2 \%$, of which $16.7 \%$ used $\mathrm{NPH}$ insulin, $14.8 \%$ used a combination of $\mathrm{NPH}$ and regular insulin, and 3.8\% used other combinations. Table also shows that at $\mathrm{P} 12$, of the $40.7 \%$ of the patients using insulin, $18.5 \%$ used NPH insulin, $16.7 \%$ used a combination of NPH and regular insulin, and $5.7 \%$ used other combinations.

To make adjustments regarding the drug therapy, the patients' economic and financial conditions were taken into consideration. It should be emphasized that most diabetic patients $(90.7 \%)$ were followed at public health care facilities, which, by determination of the Health Ministry (Regulation 371/GM), provide their drugs, that is, Biguanides, Sulfonylureas, NPH insulin, and regular insulin.

Regarding the drug therapy with oral hypoglycemic agents, it is observed there was an increase in the number of patients taking Biguanides as a monotherapy and patients taking Biguanides combined with Sulfonylureas. Concerning insulin, there was a reduction in the number of patients using NPH insulin as a monotherapy, and an increase in the use of NPH insulin combined with regular insulin.

A study by the Diabetes Control and Complication Trial Research Group ${ }^{(7)}$ evidenced that, in intensive diabetes treatments, one of the risks faced by health care professionals are the hypoglycemia episodes. These episodes occur, mainly, when insulin and oral hypoglycemic agents are used, like Sulfonylureas. In this view, concerning the occurrence of hypoglycemia episodes, before and after implementing the SDM protocol, it is observed that $50 \%$ of the 54 studied patients reported hypoglycemia episodes before beginning the treatment with the SDM protocol, and $59.3 \%$ after beginning the treatment.

\section{DISCUSSION}

Considering the aspects regarding compliance with the diet plan, it is observed that, at $\mathrm{P} 0,61.1 \%$ of the patients reported they followed the plan. At P12, this percentage rose to $92.6 \%$ patients, who reported their compliance with the diet plan created by the nutritionist at the center for research and continuing education.

On the other hand, several studies have reported low rates of patient compliance to the recommended diet plan, considering that eating is a very complex action and does not mean the mere ingestion of nutrients. Rather, it also involves a wide range of feelings and specific socio-cultural values ${ }^{(8-11)}$.

It appears that results regarding the diet plan may be related to several factors, like the fact that the center is a place in which diabetic patients were able to exchange experiences among people with similar health conditions, age, and social levels. On the other hand, there was a direct communication between patients and the

Table 1 - Numeric evolution and percentage of patients assisted at the Center for Research and Continuing Education, per treatment for diabetes mellitus. Ribeirao Preto, Sao Paulo, 2004-2005.

\begin{tabular}{|c|c|c|c|c|c|c|c|}
\hline \multirow{2}{*}{\multicolumn{2}{|c|}{ Treatment for Diabetes Mellitus }} & \multicolumn{2}{|c|}{ P0 } & \multicolumn{2}{|c|}{ P6 } & \multicolumn{2}{|c|}{ P12 } \\
\hline & & $\mathbf{N}$ & $\%$ & $\mathbf{N}$ & $\%$ & $\mathbf{N}$ & $\%$ \\
\hline \multirow{2}{*}{ Diet plan } & Yes & 33 & 61.1 & 41 & 75.9 & 50 & 92.6 \\
\hline & No & 21 & 38.9 & 13 & 24.1 & 4 & 7.4 \\
\hline Total & & 54 & 100 & 54 & 100 & 54 & 100 \\
\hline \multirow[t]{2}{*}{ Physical Activity } & Yes & 31 & 57.4 & 33 & 61.1 & 36 & 66.7 \\
\hline & No & 23 & 42.6 & 21 & 38.9 & 18 & 33.3 \\
\hline \multirow[t]{2}{*}{ Total } & & 54 & 100 & 54 & 100 & 54 & 100 \\
\hline & Biguanides as monotherapy & 13 & 24.1 & 17 & 31.5 & 19 & 35.2 \\
\hline \multirow{4}{*}{ Oral Hypoglycemic agents } & Combination of Sulfonylureas + Biguanides & 11 & 20.4 & 11 & 20.4 & 14 & 25.9 \\
\hline & Sulfonylureas as monotherapy & 7 & 13.0 & 5 & 9.3 & 2 & 3.7 \\
\hline & Others* & 4 & 7.4 & 5 & 9.3 & 6 & 11.1 \\
\hline & None & 19 & 35.2 & 16 & 29.6 & 15 & 27.7 \\
\hline \multirow[t]{2}{*}{ Total } & & 54 & 100 & 54 & 100 & 54 & 100 \\
\hline & NPH Insulin as monotherapy & 15 & 27.8 & 9 & 16.7 & 10 & 18.5 \\
\hline \multirow[t]{3}{*}{ Insulin } & $\begin{array}{l}\text { Combination of NPH Insulin + Regular } \\
\text { Insulin }\end{array}$ & 2 & 3.7 & 8 & 14.8 & 9 & 16.7 \\
\hline & Others** & - & - & 2 & 3.7 & 3 & 5.6 \\
\hline & None & 37 & 68.5 & 35 & 64.8 & 32 & 59.3 \\
\hline Total & & 54 & 100 & 54 & 100 & 54 & 100 \\
\hline
\end{tabular}

* Combination of Biguanides + Thiazolidinediones; Combination of Thiazolidinediones + Meglitinides; combination de Sulfonylureas

+ Alfa Glycosidase inhibitor; combination of Biguanides + Alfa Glycosidase inhibitor and combination of Sulfonylureas + Biguanides

+ Alfa Glycosidase inhibitor

${ }^{* *}$ Combination of NPH insulin + Lispro; combination of NPH insulin + Aspart; Insulin Glargine 
multiprofessional team; patients reported their needs were addressed, whether of psychosocial origin or associated with their personal health condition. Another factor that could have influenced patient compliance with the prescribed diet plan is their participation in creating the diet plan, which took their individuality and food preferences into consideration. It is important to emphasize that staff preparation and the simplified language used to approach patients during the implementation of the protocol could have favored the results obtained in this study.

In terms of physical activity, there was an increase in the number of patients that did any physical activity, from $57.4 \%$ at $\mathrm{P} 0$ to $66.7 \%$ at $\mathrm{P} 12$.

Exercise is an indispensable activity in the diabetes treatment as well as to prevent type 2 diabetes. Physical activity has been considered beneficial in treating diabetic patients. Healthy individuals, just as diabetic patients, present an increase in peripheral glucose use, associated with an increase in insulin action, which persists for 12 hours or more, after completing the activity ${ }^{(12)}$.

Considering the reported information and the benefits provided by exercise, the multiprofessional team was concerned with the results regarding physical activity reported at P0, P6, and P12. However, further studies are needed to verify whether variables like age and the presence of certain illnesses, such as arthrosis, fibromyalgia, neuropathies, and cardiovascular problems could be a hindrance to performing physical activities.

On the other hand, it should be considered that there are, indeed, difficulties concerning patients' compliance with physical activities and include them in their daily routine, especially when the activity is performed without the supervision of a health care professional ${ }^{(13)}$. Furthermore, not having a physical education professional in the multiprofessional team in this study was one of the factors that contributed to the obtained results.

Concerning diabetic patients' use of oral hypoglycemic agents, it is observed that, at $\mathrm{P} 0$, the most commonly used drugs were Biguanides as monotherapy $(24.1 \%)$ and Sulfonylureas combined with Biguanides (20.4\%). It is observed that, at P12, 35.2\% of the patients took Biguanides, and $25.9 \%$ took Sulfonylureas combined with Biguanides.

It is to be stressed that some patients complained about Biguanides. Complaints were related to the size of the pill and resulting gastrointestinal discomforts. It should be emphasized that the different strategies used by the multiprofessional team, like individual and group intervention, enabled patients to verbalize their difficulties, which could have contributed to the obtained results concerning accepting the oral hypoglycemia agent Biguanide.

Regarding insulin use, it is observed that, at $\mathrm{P} 0,31.5 \%$ of the patients used insulin: $27.8 \%$ used NPH insulin as monotherapy and $3.7 \%$ used it in combination with regular insulin. At P12, 40.7\% of the patients used insulin; $16.7 \%$ used NPH insulin in combination with regular insulin.

Insulin is the indicated drug for type 1 diabetes patients. For type 2 diabetes patients, it is indicated when they do not achieve good glycemic control despite following the diet plan, performing physical activity, and taking oral hypoglycemia agents at maximum doses ${ }^{(1,14-17)}$.

Insulin is indicated as a monotherapy or is taken in combination, that is, one single type of insulin can be indicated or several types can be combined as the drug therapy for type 1 and type 2 diabetes patients. Insulin can also be combined with oral hypoglycemia agents. This type of therapy is indicated exclusively upon a thorough examination of the patient's health conditions, due to the risks of hypoglycemia and weight gain ${ }^{(1,14-17)}$.

On the other side, the United Kingdom Prospective Diabetes Study Group ${ }^{(18)}$ has shown that intensive insulin therapy improves glycemia control, and thus reduces Hemoglobin A1c. Consequently, it avoids long-term microvascular complications and the risk of cardiovascular diseases, despite the hypoglycemia episodes. In this view, a broad coverage was made regarding patients who needed insulin. Therefore, $9.3 \%$ of the patients started treatment with insulin as monotherapy, and $18.5 \%$ used insulin combinations. It should be emphasized that doses as well as the number of daily insulin shots were increased.

Despite the benefits of the insulin therapy to diabetic patients, the population's taboos and beliefs about introducing insulin in these patients' treatment should be taken into consideration. On the other hand, the indication of several insulin shots sometimes restricts patients' daily activities, and poses difficulties concerning transporting the insulin as well as the public acknowledgment as a person with diabetes ${ }^{(19)}$.

In this study, patients had the opportunity of becoming an active agent in selecting their treatment. First, they were instructed about the indication of each drug, as well as their advantages and disadvantages. Other aspects were addressed, like acceptance, resistance, taboos, and prejudice against diabetes. Of the patients that started treatment with insulin $(9.3 \%)$, some $(7.4 \%)$ initially did not agree to follow it. Hence, a group approach was performed, in which psychologists helped to clarify the difficulties presented by the patients, like being afraid of the shots, of blindness, of gaining weights, and, also, the fear of needles. After the clarifications, 3.7\% promptly agreed to start the insulin therapy on the same day, whereas another $3.7 \%$ preferred to think better about the issue. It is to be emphasized that $1.9 \%$ of the patients agreed to start using insulin due to surgery, since the procedure was conditioned to glycemic control.

The multiprofessional team faced a great challenge 
with patients who chose to postpone the insulin therapy, since they had high glycemia levels. The adopted strategy consisted in approaching the issue as a group, with other patients, insulin users, who were invited to talk about their experiences. This experience exchange was enriching in terms of sensitizing diabetic patients, showing how the in-group reports positively affect the change in behavior, regarding their agreeing to use insulin.

Regarding hypoglycemia episodes, before and after implementing the SDM protocol, it is observed that of the 54 studied patients, 50\% reported hypoglycemia episodes before starting the treatment with the SDM protocol, and $59.3 \%$ after beginning the treatment.

Randomized prospective clinical studies ${ }^{(7,18)}$ have identified that intensive glycemic control increases risks for hypoglycemia. However, the benefits of glycemic control are more significant than the risks presented in hypoglycemia episodes. Therefore, it is recommended that intensive glycemic control be performed carefully and under rigorous control.

In the present study, due to changes made in the drug therapy, especially insulin and Sulfonylureas, several precautions were taken with a view to reduce risks that could cause hypoglycemia episodes. First, patients actively participated in choosing their treatment. Hence, all the advantages and disadvantages concerning each option were discussed.

Later, the orientations were strengthened to identify hypoglycemia alert signs and symptoms, as well as on how to make decisions in face of hypoglycemia episodes at home. A permanent communication channel was established, through telephone, between patients and researchers to clarify any doubts they could have and to make any changes to the treatment, if necessary.

It is important to emphasize that, during the study, it was verified it is important to establish this communication with patients to offer them the support regarding complying with the proposed treatment. This means was used to clarify doubt concerning side effects caused by the drugs, and the signs and symptoms originated by achieving the glycemic control goals, since these issues could cause patients to suspend the prescribed drug, or they could interpret these signs and symptoms as poor metabolic control.

This study also made it possible to understand the patients' treatment abandonment. It should be stressed that in the pre- and post- nursing appointment, as well as in the medical appointment, the researchers were careful to provide patients with individual orientation regarding the type, dose, times, and the side effects of the prescribed drugs. However, when they arrived home, many patients still felt they needed to use the telephone to clarify doubts. This shows the complexity of the process.

Another study finding concerns patients' knowledge about their disease. It is recognized that the knowledge described in scientific literature suggests health care professionals should make the clinical decisions regarding diabetes treatment. However, each patient's characteristics should be considered, in terms of their complaints regarding side effects, which are often left unstated in scientific literature and are underreported by other patients. The presence of hypoglycemia signs and symptoms, within good glycemic control parameters, has also been verified.

It is important to stress the difference between knowledge and information. Literature states that knowledge is more than reproducing information, since it presupposes changes in attitudes, behaviors, and life habits ${ }^{(20)}$.

On the other hand, when diabetic patients realize that the multiprofessional team is prepared for providing health care, they feel confident with the treatment and establish bonds that make them recognize the difficulties they face every day to control diabetes ${ }^{(21)}$.

\section{CONCLUSION}

The use of SDM Protocol by a multiprofessional health team increased drug coverage in diabetes treatment, as well as diabetic patient compliance with diet plans and physical activity. The present study results, as well as the experience with diabetic patients, strengthen the conviction regarding the need for continuous guidance and reinforcement for every diabetic patient, including those with a good metabolic control. It was verified that compliance to physical activity was rather low. There are few intervention studies with elderly populations with type 2 diabetes in Brazilian literature. Furthermore, the innovative character offered by psychology in the use of the protocol comprises the originality in this study.

In this view, it is essential to recognize the importance of the following issues: human resource preparation for the development of diabetes education program in health institutions; establishing a permanent means of communication between health care professionals and patients, mainly in the initial and/or adjustment phase in diabetes treatment; implement the SDM protocol in other health institutions with a view to systemize assistance and increase treatment compliance.

\section{REFERENCES}

1. 17th International Diabetes Federation Congress. Mexico City, Mexico. November 5-10, 2000. Abstracts. Diabetes Res Clin Pract. 2000; 50 Suppl 1:S1-474

2. Organização Pan-Americana da Saúde. Carmen: iniciativa para a prevenção integrada de doenças não-transmissíveis 
nas Américas. Brasília: OPS; 2003. 32 p.

3. Brown SA. Interventions to promote diabetes selfmanagement: state of the science. Diabetes Educ. 1999;25(6 Supl):52-61.

4. Rickheim P, Flader J, Carstensen K. Type 2 diabetes basics: a complete curriculum for diabetes education. Minneapolis: International Diabetes Center; 2000.

5. Tomky D, Weaver T, Mulcahy K, Peeples M. Diabetes education outcomes: what educators are doing. Diabetes Educ. 2000;26(6):951-4.

6. Mazze RS, Simonson G, Strok E, Bergenstal R, Idrogo M, Ramirez S, Hsu S; International SDM Study Group. Staged diabetes management: a systematic evidence-based approach to the prevention and treatment of diabetes and its comorbidities. Pract Diab Int. 2001; 8(7 Suppl):S1-S16.

7. The effect of intensive treatment of diabetes on the development and progression of long-term complications in insulin-dependent diabetes mellitus. The Diabetes Control and Complications Trial Research Group. N Engl J Med. 1993;329(14):977-86.

8. Péres DS, Franco LJ, Santos MA. Comportamento alimentar em mulheres portadoras de diabetes tipo 2. Rev Saúde Pública. 2006;40(2):310-7.

9. Cabrera Pivaral CE, Novoa Menchaca A, Centeno López NM. Conocimientos, actitudes y prácticas dietéticas en pacientes con diabetes mellitus II. Salud Pública Méx. 1991;33(2):166-72.

10. Cabrera Pivaral CE, Martínez Ramírez A, Vega Lóz MG, González Pérez G, Muñoz de la Torre A. Prácticas nutricias en pacientes diabéticos tipo II en el primer nivel de atención. Instituto Mexicano del Seguro Social (IMSS), Jalisco, México. Cad Saúde Pública. 1996; 12(4):525-30.

11. Williamson AR, Hunt AE, Pope JF, Tolman NM. Recommendations of dietitians for overcoming barriers to dietary adherence in individuals with diabetes. Diabetes Educ. 2000; 26(2):272-9.

12. Tuomilehto J, Lindström J, Eriksson JG, Valle TT,
Hämäläinen H, Ilanne-Parikka P, Keinänen-Kiukaanniemi S, Laakso M, Louheranta A, Rastas M, Salminen V, Uusitupa M; Finnish Diabetes Prevention Study Group. Prevention of type 2 diabetes mellitus by changes in lifestyle among subjects with impaired glucose tolerance. N Engl J Med. 2001; 344(18):1343-50.

13. Tudor-Locke C, Bell RC, Myers AM, Harris SB, Ecclestone NA, Lauzon N, Rodger NW. Controlled outcome evaluation of the First Step Program: a daily physical activity intervention for individuals with type II diabetes. Int J Obes Relat Metab Disord. 2004;28(1):113-9.

14. Grupo de Estudio de la Diabetes en la Atención Primaria de Salud - GEDAPS. Guía para el tratamiento de la diabetes tipo 2 en la atención primaria. 3a ed. Barcelona: Harcourt; 2000.

15. Davidson MB. Diabetes mellitus: diagnóstico e tratamento. 4a ed. Rio de Janeiro: Revinter; 2001.

16. Brasil. Ministério da Saúde. Secretaria de Políticas de Saúde. Plano de reorganização da atenção à hipertensão arterial e ao diabetes mellitus. Manual de hipertensão arterial e diabetes mellitus. Brasília (DF): Ministério da Saúde; 2002.

17. American Diabetes Association. Padronização de cuidados médicos em diabetes. Diabetes Care. 2004;3(2):64-84.

18. Intensive blood-glucose control with sulphonylureas or insulin compared with conventional treatment and risk of complications in patients with type 2 diabetes (UKPDS 33). UK Prospective Diabetes Study (UKPDS) Group. Lancet. 1998; 352(9131):837-53.

19. Santana MG. O corpo do ser diabético: significados e subjetividades [tese]. Florianópolis: Universidade Federal de Santa Catarina; 1998.

20. Norris SL, Engelgau MM, Narayan KM. Effectiveness of self-management training in type 2 diabetes: a systematic review of randomized controlled trials. Diabetes Care. 2001; 24(3):561-87.

21. Santos ECB, Zanetti ML, Otero LM, Santos MA. O cuidado sob a ótica do paciente diabético e de seu principal cuidador. Rev Latinoam Enfermagem. 2005;13(3):397-406. 